\title{
Nanostructures and Super-Hydrophobic Properties on the Leaves of an Indigenous Australian Plant Eucalyptus Pleurocarpa
}

\author{
Gérrard Eddy Jai Poinern, Trent Kennedy and Derek Fawcett \\ Department of Physics, Energy Studies and Nanotechnology \\ School of Engineering and Energy, Murdoch University, Murdoch, Western Australia, Australia
}

Correspondence should be addressed to: Gérrard Eddy Jai Poinern; g.poinern@murdoch.edu.au

Received date: 5 june 2014; Accepted date: 8 july 2014; Published date: 28 October 2014

Academic Editor: Hoon Eui Jeong

Copyright (C) 2014. Gérrard Eddy Jai Poinern, Trent Kennedy and Derek Fawcett. Distributed under Creative Commons CC-BY 3.0

\begin{abstract}
This study presents the results of a topographical survey of the surface features found on the leaves of an indigenous Australian plant Eucalyptus pleurocarpa (Tallerack). Field emission scanning electron microscopy was used to examine the size and morphology of various micrometer and nanometre scale features presented on the leaf surface. In particular, the features formed by the epicuticular waxes were investigated and quantified. Analysis of water contact angle measurements carried out on the adaxial surface indicated that the leaf surface was super-hydrophobic $\left(158.00 \pm 4.30^{\circ}\right)$, while the abaxial surface was found to be hydrophobic $\left(150.20 \pm 3.90^{\circ}\right)$. Microscopy examination revealed that the leaf surfaces contained an array of stomata surrounded by a rugged surface region dominated by a rim and bumps. The stomata rims and surface bumps surrounding the stomata were adorned with nanometre scale pillar structures. On the adaxial surface the mean diameter of these pillar structures was estimated to be $300 \pm 50 \mathrm{~nm}$ and lengths ranging from 1 to $7 \mu \mathrm{m}$. While the self-cleaning experiments demonstrated that the Tallerack leaf could be effectively cleaned using a fine spray of water droplets that rolled over the surface picking up both hydrophilic (Ballotini microspheres) and hydrophobic (carbon black toner) contaminants.
\end{abstract}

Keywords: nanostructures, super-hydrophobic, self-cleaning

Cite this Article as: Gérrard Eddy Jai Poinern, Trent Kennedy and Derek Fawcett (2014), " Nanostructures and Super-Hydrophobic Properties on the Leaves of an Indigenous Australian Plant Eucalyptus Pleurocarpa", Journal of Research in Nanotechnology , Vol. 2014 (2014), Article ID 339533, DOI: 10.5171/2014.339533 


\section{Introduction}

Nature, through millions of years of evolution has enabled many species to adapt to their ever-changing environment. For example, many aquatic species of flora such as the lotus (Nelumba nucifera) have evolved leaves with surface features at both the micrometer and nanometre scales, which have given the leaves both super-hydrophobic and selfcleaning properties. These properties are not unique to the lotus, in fact similar surface features and properties can be found in a variety of plants such as Indian watercress, (Trapaeolum majus) and taro, (Colocasia esculenta) leaves (Barthlott and Neinhuis, 1997A). The discovery of the Lotus effect by Barthlott \& Neinhuis highlighted the importance of surface wetting of solid surfaces not only in nature (Barthlott and Neinhuis, 1997B), but also in a variety of construction and industrial applications (Qian and Shen, 2005; Zhang et al, 2008). Studies of various features found on the surface of Lotus leaves have revealed an interesting interaction between the micrometre scale surface structures and the nanometre scale wax crystal formations. The interplay between the micrometre and nanometre scale features results in a significant increase in both surface roughness and hydrophobic nature of the leaf surface (Barthlott and Neinhuis, 1997B; Furstner and Barthlott, 2005). Because of this interplay water drops do not spread over and wet the leaf surface, but instead form spherical bead, which can easily roll off the leaf. It is during bead rolling that foreign particles are removed from the leaf surface and in the process gives the lotus leaf its selfcleaning properties. Determination of surface wettability is derived by measuring the internal contact angle of a water droplet in contact with a surface. Measurements of internal contact angle of water drops on smooth flat surfaces are usually less than $90^{\circ}$, while smaller the contact angles indicate increasing surface wetting. Whereas larger contacts angles, normally between $90^{\circ}$ and $150^{\circ}$, indicate the surface is becoming more non-wetting or hydrophobic, and as a result the water drop becomes more spherical. Moreover, when the water contact angle exceeds $150^{\circ}$, the surface becomes superhydrophobic and this is indeed the case of the lotus leaf, which has a contact angle of 162 (Barthlott and Neinhuis, 1997B).

Recently, Poinern et al. examined the superhydrophobic nature produced by nanometre scale structures found on the leaf surface of Eucalyptus macrocarpa or Mottlecah. But unlike the lotus leaf, which lives in an aquatic environment, the Mottlecah lives in the semiarid southwest region of Australia (Poinern et al, 2011) . The term eucalyptus is derived from the Greek term meaning "well covered" and describes a diverse genus of flora predominately found in the Australian continent. The genus forms a large part of the Australian landscape forming 95 percent of the indigenous woodlands. While the majority of the over 700 different species are native to Australia, several species have been found in New Guinea, Indonesia and as far north as the Philippine islands. Moreover, several species have been introduced to other dry regions found in the Mediterranean, Africa, India, China and California (Pryor, 2014; Brooker and Kleinig, 2001).

Eucalyptus pleurocarpa, a member of the mallee subgroup of Eucalypts, is found along the sand plains north of Perth, extending through parts of the eastern wheat belt down to Esperance in Western Australia as seen in Figure 1 (b). Both locations are relatively dry for coastal regions, with each receiving around 300 to $500 \mathrm{~mm}$ of rainfall annually (Bureau of Meteorology, 2014). The eucalypt is commonly known as the Tallerack, but it has also been known by a number of different names such as Mealy Gum, Silver Marlock, White Marlock, Leaved Marlock and Eucalyptus tetragona. The plant can reach a height of $4 \mathrm{~m}$, while its bark tends to be smooth and ranges in colour from a pale grey to a deep grey-brown colour. The leaves are broad, rounded and grey-blue in colour. The 
stems of juvenile plants tend to be square in shape, smooth and covered by a silverywhite powdery bloom on branches, leaves, buds and fruits. When in bloom, the flowers are white and appear in groups of three, while the fruit appears cubic in shape (ASGAP, 2014). The plant is generally identified by the four directions in which its fruits and leaves extend from the branches as seen in Figure 1 (a). However, it's the silverywhite appearance of the leaves that suggests the presence of some interesting surface features and the ability of the leaves to maintain their clean appearance.

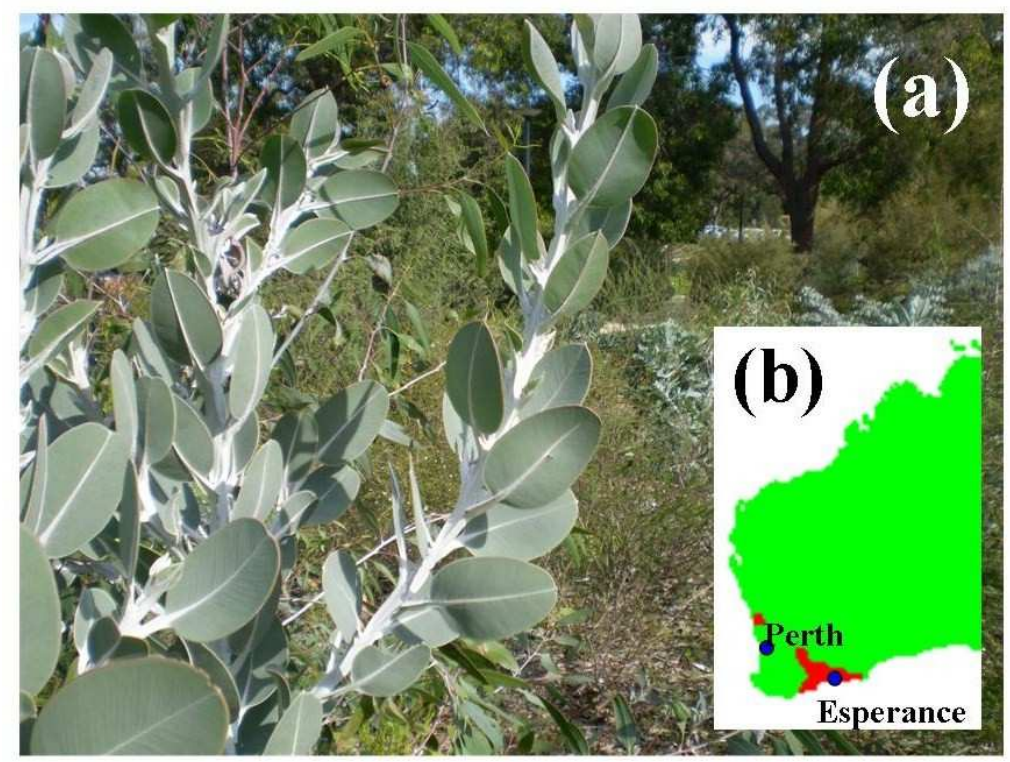

Figure 1 : (a) Eucalyptus pleurocarpa (Tallerack) a plant native to South Western Australian (b) Regional locations of the Tallerack

In this study, the size, shape and morphology of surface features found on the cuticle layer of the Tallerack leaf was examined using field emission scanning electron microscopy (FESEM). The cuticle layer is composed of a cutin matrix, waxes embedded in, (intracuticular) and deposited on, (epicuticular) the surface of the matrix (Dominguez et al, 2011). The cuticle waxes consist of a complex mixture of straightchain aliphatic hydrocarbons and a variety of other compounds such as secondary alcohols and $\beta$-diketones that forms the characteristic waxy appearance of the leaves (Storey and Price, 1999). The structure and composition of various leaf cuticle types have been extensively reviewed in the literature and the reader is referred to these articles for further details (Bargel et al, 2006; Jeffree, 1996; Walton, 1990). The function of the epicuticular waxes is to form a three dimensional matrix across the leaf surface which scatters UV and blue light radiation that lowers leaf temperatures, which in turn reduces water loss during transpiration and permits the exchange of gases used in photosynthetic processes (Eglinton and Hamilton, 1967). Moreover, it is the composition, crystallinity and physical structure of the epicuticular waxes that contribute to the wetting properties of the leaf surface. In this study, the authors have focused on examining and quantifying the hierarchal structures presented on the 
surface of the Tallerack leaf. Furthermore, the self-cleaning properties of the leaf surface were investigated using contaminants such as carbon black (hydrophobic material) and Ballotini (sodalime glass) microspheres (hydrophilic material) (Potters Europe, 2014).

\section{Materials and Methods}

\section{Leaf Samples}

A wide selection of healthy leaves were collected from various plant locations (top, north, south, east, and west), with an average of 10 leaves per location and leaves ranging from young to mature being harvested. Dyed water droplets ranging in size from 10 to 15 $\mu \mathrm{L}$ composed of Milli- ${ }^{\circledR}$ water [Barnstead Ultrapure Water System D11931; Thermo Scientific, Dubuque, IA (18.3 $\mathrm{M} \Omega \mathrm{cm}-1)]$ and commercially available water based food dyes were deposited from a syringe in four different positions on each leaf side to ensure that the drops were not sitting on a contaminated or damaged part of the leaf. The coloured droplets provided the initial conformation of the hydrophobic behaviour of the leaf surfaces as seen in Figure 2 (a).

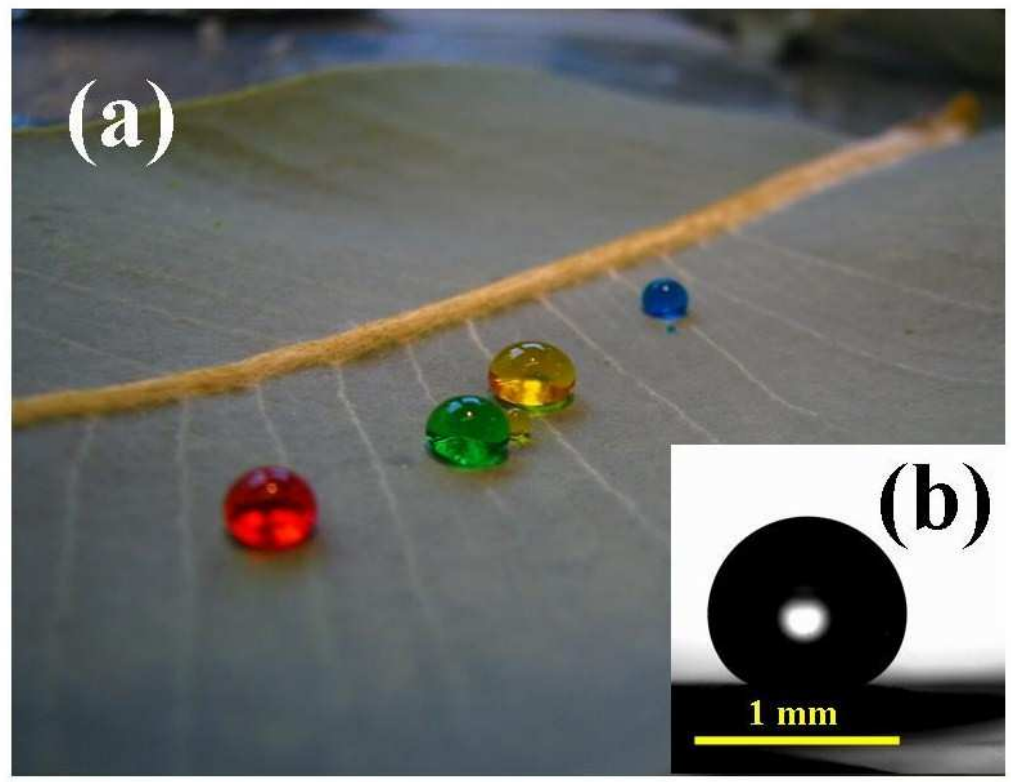

Figure 2: (a) Dye-coloured water droplets, with high contact angle resting on a Tallerack leaf (b) Image of a sessile water drop used during contact angle measurements

\section{Contact Angle Measurements}

The static of the contact angle (CA) measurements were performed using a CAM 100 system from KSV Instruments Inc (Biolin Scientific, Helsinki, Finland). Images of each droplet were taken at a rate of one per second for 4 seconds to reduce any influence of oscillations. Depending on leaf size, a selection of various locations over both the adaxial (top) and abaxial (bottom) sides were used for CA measurements. On average, six sample locations, each with four water droplets, were studied on each side of the leaf. CA measurements were taken of each of the four droplets, with an average of 24 droplets being examined on each side of the leaf. Images with the most comparable angle 
measurements of the left and right hand sides of each droplet were used to calculate the CA. A typical image of a sessile water drop used during contact angle measurements is presented in Figure 2 (b). From these measurements the mean and standard deviation for the static CA were subsequently calculated for both sides of the leaf.

\section{Field Emission Scanning Electron Microscopy (FESEM) Measurements}

The FESEM micrographs were taken using a Zeiss 1555 VP-FESEM (Carl Zeiss MicroImaging $\mathrm{GmbH}$, Gottingen, Germany) electron microscope. Leaf samples were dried in a vacuum oven (Napco 5831 Vacuum Oven; Precision Scientific, Inc) to reduce their water content. Samples were mounted on individual substrate holders using carbon adhesive tape before being sputter coated with a $2 \mathrm{~nm}$ layer of gold to prevent charge build up using a Cressington 208HR High Resolution Sputter coater. The micrographs were then used to investigate the surface features and morphologies of both the adaxial and abaxial sides of the Tallerack leaf samples.

\section{Self-cleaning Experiments}

Two sets of self-cleaning experiments were carried out. The first contaminant examined was Ballotini microspheres, which is a hydrophilic material. The second contaminant examined was carbon black toner, which is a hydrophobic material. In the first case, Ballotini microspheres, which are composed of soda-lime glass, were milled to produce a fine powder (Potters Europe, 2014). The resulting powder was then finely sprinkled over the entire leaf surface with any excess powder being shaken off. The leaf was then oriented with a slight dipping angle relative to the horizontal plane and a $10 \mu \mathrm{L}$ droplet of Milli- ${ }^{\circledR}{ }^{\circledR}$ water was placed onto the top left hand portion of the leaf surface using a glass pipette fitted with a rubber bulb. The droplet acting under the influence of gravity rolls across the leaf surface picking up the microspheres and leaving a clean pathway. In the second case, carbon black toner was sprinkled over a clean Tallerack leaf. Excess toner was shaken off and then the leaf was oriented with a slight dipping angle relative to the horizontal plane, so that water droplets can roll off the leaf. A number of short fine sprays of water (from a normal handheld and finger-pump garden spray mister, $1.10 \mathrm{~mL}$ of water per spray) were sprayed over the surface of the leaf. As the fine mist of water droplets rolled down the leaf they picked up, the toner formed water marbles and in the process cleaned the surface. In both cases, the motion of the water droplets was filmed using a small standard digital camera (Olympus FE220; Olympus Corp, Tokyo, Japan). The sequential frames were then extracted to show the selfcleaning effect of the leaf.

\section{Results and Discussions}

\section{Contact Angle Measurements}

Initial visual inspection of both the adaxial and abaxial sides of the leaf revealed that the adaxial surface had a more substantial coating of surface waxes. Water droplets deposited on both the adaxial and abaxial sides of the leaf revealed that both surfaces were hydrophobic as seen in Figure 2 (a). A typical image used to make CA measurements of a sessile water droplet sitting on the adaxial surface of the leaf is presented in Figure 2 (b). The image reveals that water droplets formed on the adaxial surface have a high degree of beading and pronounced spherical morphology. Analysis of CA measurements taken on the adaxial leaf surface revealed that the mean and standard deviation for the static contact angle was calculated to be $158.00 \pm 4.30^{\circ}$, which makes the top surface of the leaf super-hydrophobic. A similar analysis of the abaxial surface revealed a contact angle of $150.20 \pm 3.90^{\circ}$, which makes the bottom of the leaf hydrophobic. 


\section{FESEM Topographical Survey of Adaxial and Abaxial Leaf Surfaces}

The FESEM survey of the adaxial leaf surface reveals an undulating landscape peppered with numerous stomata as seen in Figure 3 (a). The spacing between each stomata ranges from 75 to $100 \mu \mathrm{m}$, with all stomata being surrounded by a rugged surface region dominated by a rim and bumps. The mean stomata diameter was estimated to between 20 to $30 \mu \mathrm{m}$ and the height of the bumps ranging in from 2 to $5 \mu \mathrm{m}$. The stomata rims and surface bumps surrounding the stomata
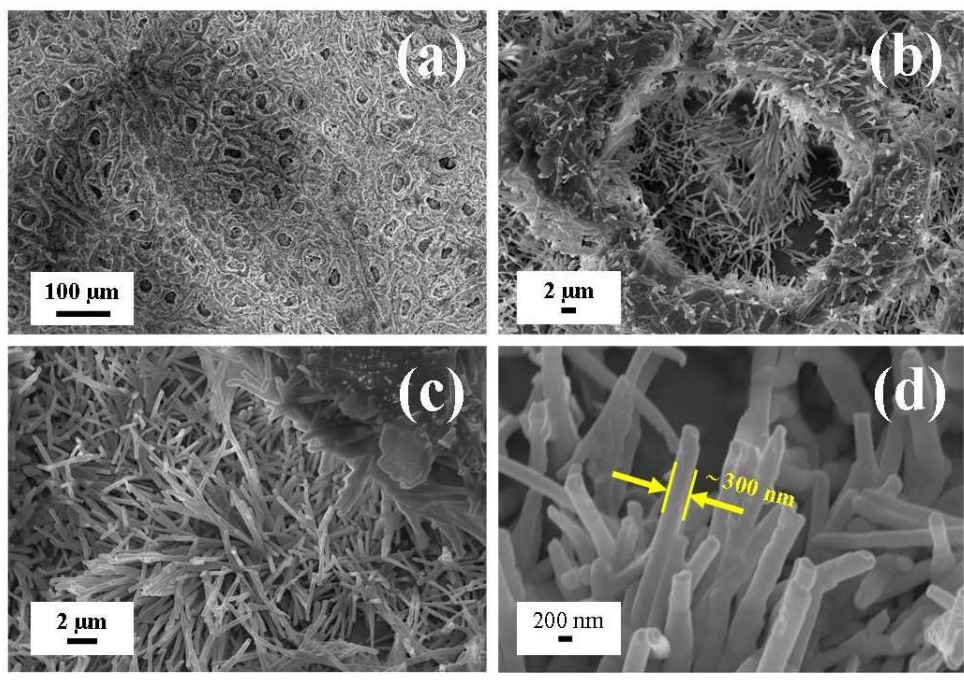

Figure 3 : FESEM topographical survey of adaxial surface: (a) typical landscape showing the presence of stomata and surface features, (b) enlarged view of single stomata.

(c) epicuticular wax nanometre pillars covering surface, (d) typical diameter of a nanometre pillar

A similar FESEM survey of the abaxial leaf surface revealed a landscape dominated by stomata with diameters ranging from 20 to $40 \mu \mathrm{m}$ and stomata spacing ranging from 70 to $100 \mu \mathrm{m}$ as seen in Figure 4 (a). The stomata rims and surface bumps surrounding the stomata are more dominant than those found on the adaxial surface and range in size from 5 to $15 \mu \mathrm{m}$. But unlike the adaxial surface, the abaxial rims and surface bumps are lightly adorned with nanometre are heavily adorned with nanometre scale pillar structures as presented in Figures 3 (b) and (c). These nanometre scale pillars are also present over the remaining leaf surface which also displays smaller bumps or rises over the surface. There is also some evidence of secondary growth with some pillars branching out from existing pillars, while other pillars show signs of splitting and forming modified pillar structures as seen in Figure 3 (c). The mean diameter of these pillar structures was estimated to be around $300 \pm 50 \mathrm{~nm}$ and lengths ranging from 1 to $7 \mu \mathrm{m}$ as seen in Figure (c) and (d). 
of secondary growth which has produced a pronounced wavy-coral, fractal-like structure. It is the differences in the abaxial epicuticular wax formed pillar structures that produced a reduced a lower hydrophobic response compared to the adaxial surface. Therefore, the key factors for producing the super-hydrophobic adaxial leaf surface was the presence of longer pillar lengths, higher pillar densities and less secondary growth leading of the epicuticular waxes.
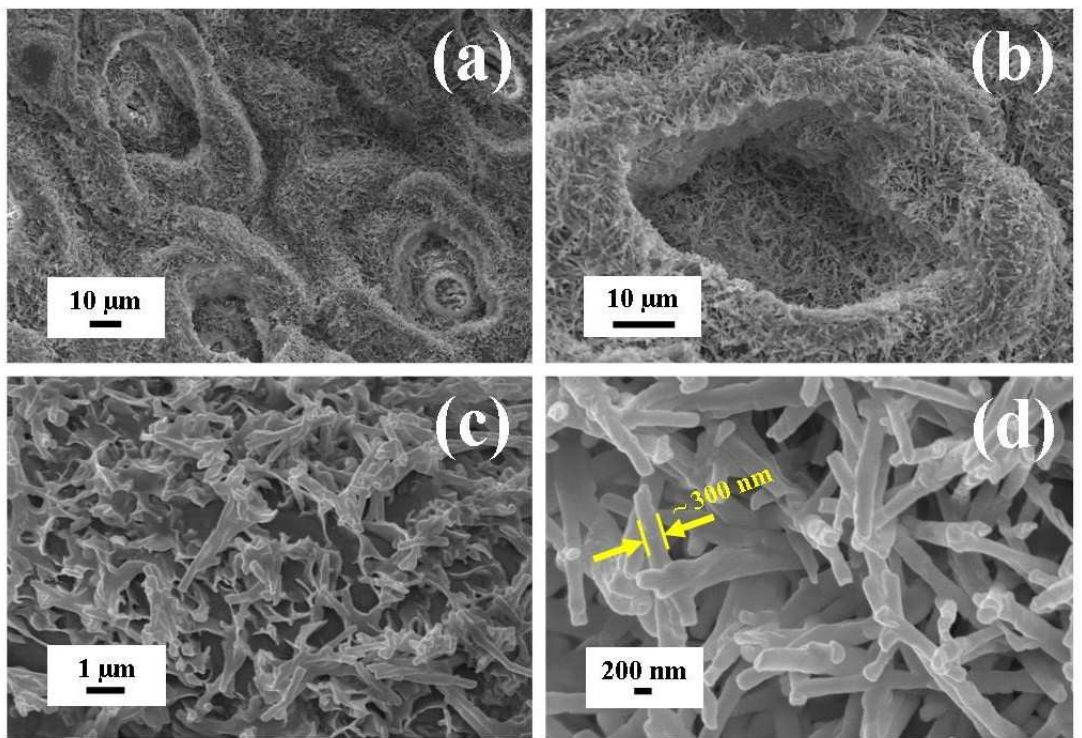

Figure 4: FESEM topographical survey of abaxial surface: (a) typical landscape with pronounced stomata (b) enlarged view of stomata showing fewer pillars (c) epicuticular wax forming a wavy-coral like structure with shorter pillar lengths (d) typical diameter of a nanometre pillar.

\section{Self Cleaning Properties of the Eucalyptus Pleurocarpa (Tallerack) Leaf}

The potential self-cleaning properties of the Tallerack leaf were investigated using two commercially available products, which formed the contaminants. The first contaminant examined was Ballotini microspheres, (hydrophilic), while the second contaminant was carbon black toner, which is a natural hydrophobic material. In the first experiment, a $10 \mu \mathrm{L}$ water droplet was deposited onto a Ballotini microsphere based powder dusted Tallerack leaf, (adaxial) using a pipette as seen in Figure 5 (a). As the water droplets rolled over the surface it immediately picked up the powder and left a clean pathway. The Ballotini spheres could be seen suspended within the droplet with no particular threshold concentration evident as seen in Figure 5 (b) and (c). 


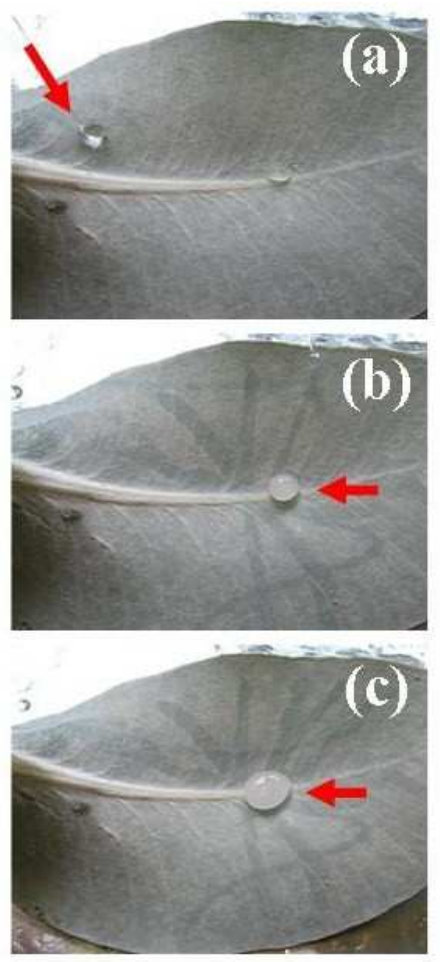

Figure 5: Sequential photographic images of a water droplet moving over the adaxial leaf surface of Eucalyptus pleurocarpa: (a) initial position of deposited water droplet (b) water droplets rolling over dusted leaf surface picking up Ballotini spheres and (c) water droplet collecting Ballotini spheres and leaving a clean pathway.

In the second self-cleaning experiment, carbon black toner (hydrophobic contaminant) was used to coat the adaxial leaf surface. In the first procedure, a $10 \mu \mathrm{L}$ water droplet was deposited onto the carbon treated leaf using a pipette as seen in Figure 6 (a). As the water droplets rolled over the leaf surface it immediately picked up the carbon powder and left a clean pathway. However, because the carbon toner is hydrophobic it tended to accumulate on the outside of the droplet and eventually covered the surface of droplet which ultimately prevented it from picking up any additional amounts of toner as seen in Figure 6 (b) and (c). In the second procedure, a carbon toner treated leaf was sprayed with water using controlled amounts of water. Each spray used $1.10 \mathrm{~mL}$ of water and only three sprays were used to completely clean the leaf surface as seen in Figure 6 (d), (e) and (f). During each spray the water droplets would roll down the leaf surface picking up carbon toner particles to form small black water marbles which roll and run-off the leaf.

The results of this study have clearly demonstrated some remarkable superhydrophobic and self-cleaning properties of the Tallerack leaf. These properties are clearly related to the micrometer and nanometre scale surface structures 
presented on the surface of the leaf. The combination of micrometer and nanometre scale features, in particular the nanometre scale pillars, present on the
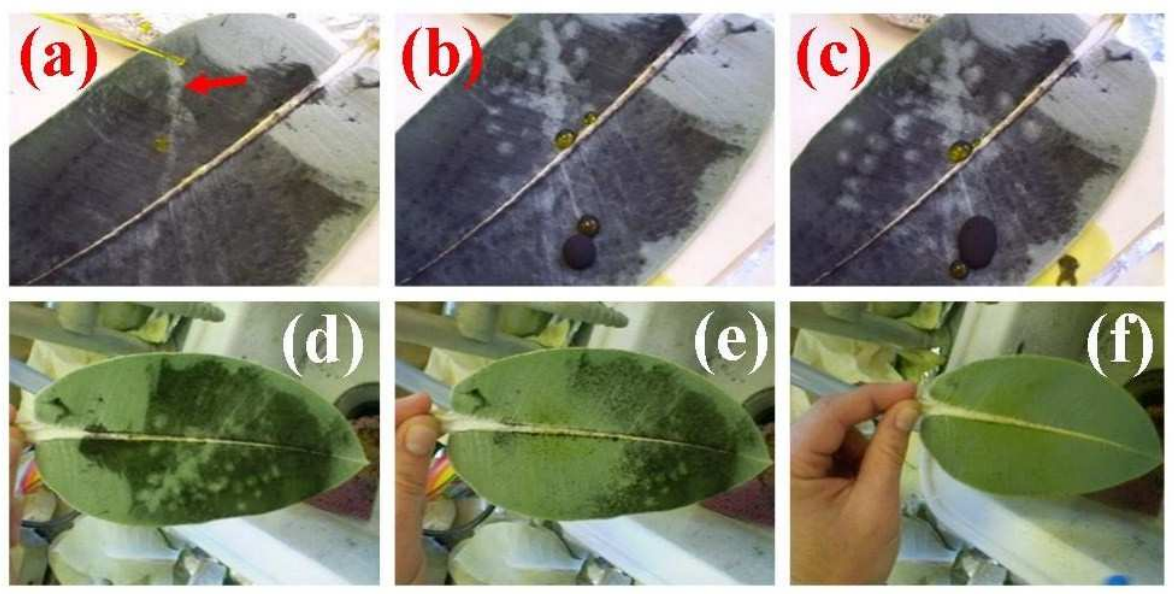

Figure 6: Photographic images of a water droplet moving over the adaxial leaf surface of Eucalyptus pleurocarpa covered by carbon black toner: (a) initial position of deposited water droplet (b) water droplets rolling over dusted leaf surface picking up carbon toner particles and (c) water droplet collecting carbon black toner particles to form rolling black marbles of water and leaving a clean pathway. Water being sprayed onto adaxial leaf surface: (d) $1^{\text {st }}$ spray (e) $2^{\text {nd }}$ spray and (f) $3^{\text {rd }}$ spray with each using $1.10 \mathrm{~mL}$ of water to completely clean leaf.

Tallerack leaf are similar in nature to those found in studies of the Eucalyptus macrocarpa [6] and the Lotus leaf $[1,2]$. The importance of this study lies in the discovery of super-hydrophobic properties in an indigenous Australian plant which has similar leaf surface features to a plant investigated in an earlier study of the Eucalyptus macrocarpa (Mottlecah) [6]. The leaf structures formed by the epicuticular waxes in both the Mottlecah and Tallerack produce nanometre scale pillars of similar size. For example, the Mottlecah leaves are covered with an array of small regular and periodic structures or bumps that have a mean diameter of $20 \mu \mathrm{m}$ and have mean spacing between centres of $26 \mu \mathrm{m}$. And importantly, the bumps are covered with pillars that have mean diameter of $280 \mathrm{~nm}$ at their tips. The pillars are around $10 \mu \mathrm{m}$ in length and protrude vertically the surface of the bump. In this study, the Tallerack leaf pillars were found to have a mean diameter of $300 \mathrm{~nm}$ and a length of $7 \mu \mathrm{m}$ on the adaxial surface. The Mottlecah's static contact angle calculated to be $162^{\circ}$, while the Tallerack's calculated contact angle was estimated to be $158^{\circ}$ (adaxial). Both leaves are superhydrophobic and produce highly spherical water droplets.

Further studies are needed to elucidate the mechanisms involved in engineering the epicuticular waxes into the micrometre and nanometre scales structures found on the surface of both the Mottlecah and Tallerack 
leaves. Because of similar features such as the pillar structures, the formation mechanism in both leaves appears similar and a common process is believed to operate. Understanding this formation process is important and could allow it to be translated to a number of potential industrial applications such as self-cleaning and water repellent textiles and surface treatments and paints; drag reduced material surfaces and antifouling surfaces.

\section{Conclusion}

This study has revealed that the leaf surface features and properties of the Tallerack's epicuticular waxes render the adaxial surface super-hydrophobic (158을 , while the abaxial surface was found to be hydrophobic $\left(150^{\circ}\right)$. Both leaf surfaces demonstrated a selfcleaning capability, but the adaxial surface had the superior self-cleaning performance. Because of the exceptional surface properties of the Tallerack leaf, in particular the selfassembly of its epicuticular waxes to form nanometre scale pillars, further research is needed to fully understand the formation mechanisms of the waxes. If this mechanism can be fully understood, modelled and effectively reproduced, it has the potential to be used in a number applications such selfcleaning coatings for buildings and water repellent treatments for textiles to name just few.

\section{Acknowledgement}

The authors would like to thank Dr ZhongTao Jiang, (WANRI Research Fellow) and Mr. Tim Thomson, (WANRI Research Assistant). Ms. Xuan Le is acknowledged for her assistance with the FESEM imaging.

\section{Disclosure}

The authors report no conflict of interest in this work.

\section{References}

1. (ASGAP, 2014), ASGAP, Association of Societies for Growing Australian Plants (2014), "Eucalyptus pleurocarpa" [online], [Retrieved January 15, 2014], http://asgap.org.au/e-ple.html

2. Bargel, H, Koch, K, Cerman Z. and Neinhuis C. (2006) "Structure-function relationships of the plant cuticle and cuticular waxes - a smart material?" Functional Plant Biology, 33 (1) 893-910.

3. Barthlott, W, and Neinhuis, C. (1997 A) "Characterization and distribution of water repellent, self-cleaning plant surfaces," Annals of Botany (London), 79 (6) 667-677.

4. Barthlott, W, and Neinhuis, C. (1997 B) "Purity of the sacred lotus, or escape from contamination in biological surfaces," Planta, 202 (1) 1-8.

5. Dominguez, E, Heredia Guerrero, J. A. and Heredia, A. (2011) "The biophysical design of plant cuticles: an overview," New Phytol, 189 (4) 938-949.

6. Brooker, M. I. H. and Kleinig, D. A. (2001) Field Guide to Eucalypts: South-Western and Southern Australia, Bloomings Books, Sydney, Australia.

7. (Bureau of Meteorology, 2014), Bureau of Meteorology: Weather Updates and Forecasts, [online]. Australian Government Website, [February 4, 2014]. Available: www.bom.gov.au

8. Eglinton, G. R. J. and Hamilton, R. J. (1967) "Leaf epicuticular waxes," Science, 156 (1) 1322-1335.

9. Furstner, R. and Barthlott, W. (2005) "Wetting and self-cleaning properties of artificial super hydrophobic surfaces," Langmuir. 21 (3) 956-961.

10. Jeffree, C. E. (1996) Structure and ontogeny of plant cuticles, In: Plant Cuticle, 
An Integrated Functional Approach, BIOS Scientific Publishers, Oxford, United Kingdom.

11. Poinern, G. E. J, Le, X. and Fawcett, D. (2011) "Super-hydrophobic nature of nanostructures on an indigenous Australian eucalyptus plant and its potential application," Nanotechnology, Science and Applications, 4 (1) 113-121.

12. (Potters Europe, 2014), Potters Europe (2014), Materials Specifications, [online], [Retrieved December 19, 2013], Available at: http://www.potterseurope.com

13. Pryor, L. D. (19810 Australian Endangered Species: Eucalypts. Special Publication: Commonwealth of Australia, Canberra, Australia.
14. Qian, B. T. and Shen, Z. Q. (2005) "Fabrication of super-hydrophobic surfaces by dislocation selective chemical etching on aluminium, copper and zinc substrates," Langmuir, 21(20) 9007-9009.

15. Storey, R. and Price, W. E. (1999) "Microstructures of the skin of d'Agen plums," Scientific Horticulture, (Amsterdam), 81(3) 279-286.

16. Walton, T. J. (1990) "Waxes, cutin and suberin," Methods Plant Biochemistry, 4 105158.

17. Zhang, X, Shi, F, Nui, J, Jiang, Y. and Wang Z. (2008) "Super hydrophobic surfaces: from structural control to functional application," Journal of. Materials Chemistry, 18 621-633. 